\title{
Parallel Hole and Rotating Slat Collimators: Comparative Study using Digital Contrast Phantoms
}

\author{
Lin Zhou, Kathleen Vunckx and Johan Nuyts
}

\begin{abstract}
The parallel hole (PH) collimator is the most commonly used collimator in single photon emission computed tomography (SPECT) imaging. A well-known limitation of the PH collimator is its low geometric efficiency. For better geometric efficiency without compromising the resolution, a rotating slat (RS) collimator is a potential alternative. In our previous work, a linear relation between the image resolution and the optimal collimator aperture was derived for both the PH and the RS collimator systems, and the two collimator systems were compared with the optimized geometries using different uniform phantoms. In this study, the $\mathbf{P H}$ and the $\mathrm{RS}$ collimator system were further compared using digital contrast phantoms. Three figures of merit (FOMs) were calculated for comparison, i.e., the contrast-to-noise ratio (CNR) in a pixel of interest, the CNR in a region of interest, and the signal-to-noise ratio (SNR) of a channelized Hotelling observer (CHO). All the FOMs were calculated analytically using an efficient approximation method.

The comparison was done in both planar imaging and volume imaging. The size of the digitized phantom was varied, resulting in a detector area coverage from $\mathbf{2 . 5} \%$ to $\mathbf{4 0} \%$. The main results are: (1) With the optimal collimator apertures, the gains (RS over PH) in all FOMs show the same trends in the system comparison. (2) The performance of the two collimators is similar for cold spot imaging with the largest phantom given in this study, while RS outperforms PH for all the other cases. (3) For system optimization, the optimal collimator apertures depend on the FOM to be optimized, however the ratio of the apertures $(\mathrm{PH} / \mathrm{RS})$ is always around $\sqrt{2}$, a value that we derived analytically in our previous work.
\end{abstract}

\section{INTRODUCTION}

One of the factors limiting the image quality in single photon emission computed tomography (SPECT) imaging is the tradeoff between the spatial resolution and the geometric efficiency. For example, a conventional parallel hole (PH) collimator, which consists of a two-dimensional array of long narrow holes, only detects photons whose trajectories are almost parallel to these holes (Fig. 1(a)). Making these holes smaller will improve the spatial resolution, but at the same time decreases the geometrical efficiency significantly. For a reasonable spatial resolution, the geometric efficiency of a $\mathrm{PH}$ collimator is always very low. In contrast, a rotating slat (RS) collimator consists of a series of long thin septa which are positioned parallel to each other and perpendicular to the detector surface (Fig. 1(b)). This configuration allows in-plane photon collection, therefore a RS collimator can achieve a much

The authors are with Medical Imaging Research Center, Dept. of Nuclear Medicine, University Hospitals Leuven \& Dept. of Imaging and Pathology, KU Leuven, 3000 Leuven, Belgium.

This work is supported by F.W.O. grant G.0569.08. and I.U.A.P. grant NIMI
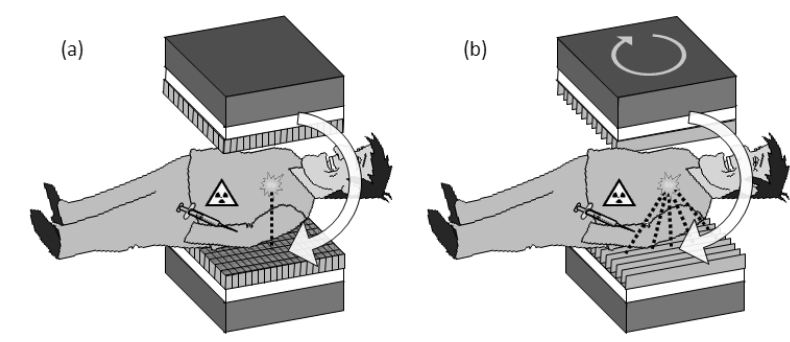

Fig. 1. (a) parallel hole ( $\mathrm{PH})$ collimator system. (b) Rotating slat (RS) collimator system.

higher geometric efficiency without any resolution loss [1]-[8]. However, in order to interpret the plane integral data acquired by RS, even for planar imaging, an extra reconstruction step is needed, resulting in increased noise propagation.

It is not obvious whether or not a higher geometric efficiency obtained by the RS collimator will lead to improved image quality. In our previous work [9], the PH and the RS collimation systems were compared by investigating the contrast-to-noise ratio (CNR) in the central point and a central region of interest (ROI) in homogeneous phantoms. In that work, we introduced the concept of the optimal collimator aperture, which leads to the maximal CNR for a given resolution in the reconstructed image [9]. The two collimator systems were first optimized and then compared using their optimized geometries. The rankings of the two collimators were in agreement with [5]-[7], but the computed performance ratios for RS versus $\mathrm{PH}$ collimation were different, since the optimal collimator apertures were not used in those studies.

In reality, the analysis of a SPECT image usually involves the observation or quantification of a hot/cold lesion or a region of interest (ROI) in a hot/cold organ. Therefore, for clinical applications, results obtained in contrast phantoms may be more clinically relevant than those obtained in perfectly uniform and spherical phantoms. In [8], a lesion contrast recovery and background noise study was performed, where the projections of the two systems were reconstructed using the rescaled blockiterative expectation maximization (RBI-EM) algorithm [10]. The contrast-to-noise analysis showed that the RS collimator leads to an improved (15-45\%) contrast recovery compared to the PH collimator for the same background noise level for both the hot and the cold lesions. However, it was not clear how much of the improvement was due to the high geometric efficiency of the RS collimator and how much was due to the better energy resolution of the new solid-state detector applied 
in the RS system. Van Holen et al. made a further investigation of this problem. They applied the same type of detector with the same dimension, and used Monte Carlo simulations for accurate system modeling. The two collimator systems were compared in planar imaging [11] and volume imaging [12], respectively, using the maximum likelihood expectation maximization (MLEM) reconstruction. The conclusion was that for planar imaging, the PH collimator only outperforms RS for cold lesions in a very large warm background, whereas in tomographic imaging, the RS always outperforms the $\mathrm{PH}$ system. The analysis was done with fixed object-to-detector distances and there was no attempt to optimize the system geometries.

In this study, the PH and the RS collimator systems were compared based on contrast phantoms, using the same approaches as in [9]. A pre-defined resolution was imposed in the reconstructed image. The CNR in pixels of interest and the $\mathrm{CNR}$ in regions of interest (ROIs) were calculated analytically. In addition, to estimate the lesion detectability, one of the analytical methods in [9] was extended to calculate the SNR of the channelized Hotelling observer (CHO), a numerical observer that has good correlation with human performance [13]-[16] for a lesion detection task. All the FOMs were estimated with the ratio of the collimator apertures (PH over RS) equal to $\sqrt{2}$, a value proposed in [9] for fair comparison of the pixel CNRs. Furthermore, the geometries of both collimator systems were also optimized to maximize each FOM, respectively.

\section{Analytical Method}

The analytical method, used for fast prediction of the CNR in the reconstruction, was first proposed by [17]-[19] for the converged maximum-a-posteriori (MAP) reconstruction. It was later adapted for post-smoothed MLEM with a pre-defined target resolution [20], [21]. For a certain pixel $j$ in the reconstructed image $\Lambda$, a set of filters, $Q_{L}^{j}$ and $Q_{C}^{j}$, are defined to enable the calculation of the local impulse response and the (co-)variance in the $j-$ th voxel [9], [20], [21]:

$$
\begin{aligned}
& Q_{L}^{j}=P^{j} G^{j} F^{j} \\
& Q_{C}^{j}=P^{j} G^{j} F^{j} G^{j T} P^{j}
\end{aligned}
$$

All the matrices are in $\mathbb{R}^{J \times J}$, with $J$ the number of pixels in the reconstructed image. $F^{j}$ is the approximated Fisher information matrix, $G^{j}$ is the approximated pseudoinverse of $F^{j}, P^{j}$ is an isotropic Gaussian post-smooth filter that tries to impose the given target resolution, and ${ }^{T}$ denotes transpose. All factors are $j$-dependent due to the assumption of local shiftinvariance [20], [21]. Details about the calculation of $F^{j}, P^{j}$ and $G^{j}$ can be found in [9].

For the chosen pixel $j$, the linearized local impulse response (LLIR) $l_{\text {pix }}$ and the covariance image $\operatorname{Cov}_{\text {pix }}$ can be approximated as:

$$
\begin{aligned}
l_{\text {pix }}(\Lambda) & \approx Q_{L}^{j} e^{j} \\
\operatorname{Cov}_{\text {pix }}(\Lambda) & \approx Q_{C}^{j} e^{j}
\end{aligned}
$$

with $e^{j}$ the $j$-th unit vector. The $j$-th elements of $l_{\text {pix }}(\Lambda)$ and $\operatorname{Cov}_{\text {pix }}(\Lambda)$ are the contrast recovery coefficient (CRC) and the variance (VAR) in pixel $j$ :

$$
\begin{aligned}
& \mathrm{CRC}_{\text {pix }} \approx e^{j T} Q_{L}^{j} e^{j} \\
& \operatorname{VAR}_{\text {pix }} \approx e^{j T} Q_{C}^{j} e^{j}
\end{aligned}
$$

For a small uniform ROI centered at pixel $j$, the vector of the ROI is written as:

$$
R^{j}(i)= \begin{cases}1 & \text { if } i \in \mathrm{ROI}, \\ 0 & \text { if } i \notin \mathrm{ROI}\end{cases}
$$

with $i$ the pixel index in the image space. The CRC and the variance of the mean value of this ROI are [21]:

$$
\begin{aligned}
\mathrm{CRC}_{\mathrm{ROI}} & \approx \frac{1}{N_{R}} R^{j T} Q_{L}^{j} R^{j} \\
\mathrm{VAR}_{\mathrm{ROI}} & \approx \frac{1}{N_{R}^{2}} R^{j T} Q_{C}^{j} R^{j}
\end{aligned}
$$

with $N_{R}$ the total number of pixels in the ROI.

The CNR in a pixel or a ROI is defined as:

$$
\begin{aligned}
\mathrm{CNR}_{\text {pix }} & =\mathrm{CRC}_{\text {pix }} / \sqrt{\mathrm{VAR}_{\text {pix }}} \\
\mathrm{CNR}_{\mathrm{ROI}} & =\mathrm{CRC}_{\mathrm{ROI}} / \sqrt{\mathrm{VAR}_{\mathrm{ROI}}}
\end{aligned}
$$

For the lesion detectability, the signal-to-noise ratio of a channelized Hotelling observer was used as a figure of merit. $\mathrm{SNR}_{\mathrm{CHO}}$ is calculated as [13]:

$$
\mathrm{SNR}_{\mathrm{CHO}}=\sqrt{z^{T} U^{T} K^{-1} U z}
$$

with $z$ the ensemble mean difference of a reconstruction with and without the lesion, $U$ the frequency-selective channels that mimic the human visual system, and $K$ the covariance matrix of the channel output. For small lesions, we assumed that the presence of the lesion has negligible effect on the data. For a lesion located near pixel $j$, the approximations for $z$ and $K$ can be derived based on [20], [22]-[24]:

$$
\begin{aligned}
z & \approx Q_{L}^{j} \bar{f}_{l} \\
K & \approx U Q_{C}^{j} U^{T}
\end{aligned}
$$

with $\bar{f}_{l}$ the expectation of the lesion profile.

The analytical approximations can be validated by doing post-smoothed MLEM reconstructions with multiple noise realizations. Given a certain pixel $j, \mathrm{CRC}_{\mathrm{pix}}, \mathrm{CRC}_{\mathrm{ROI}}$ and $z$ can be estimated from the reconstructions of the noiseless projection data $^{1}$ of the phantom with and without an impulse, a ROI or a lesion located/centered at $j$. $\mathrm{VAR}_{\text {pix }}, \mathrm{VAR}_{\mathrm{ROI}}$ and $K$ can be calculated from the reconstructions of projection data with multiple noise realizations ${ }^{2}$.

\footnotetext{
${ }^{1}$ Here it is assumed that the ensemble mean of the reconstructions is approximately equal to the noiseless reconstruction [18].

${ }^{2}$ The relative error on the standard deviation is $\sqrt{1 / 2(N-1)}$, with $N$ the number of noise realizations.
} 


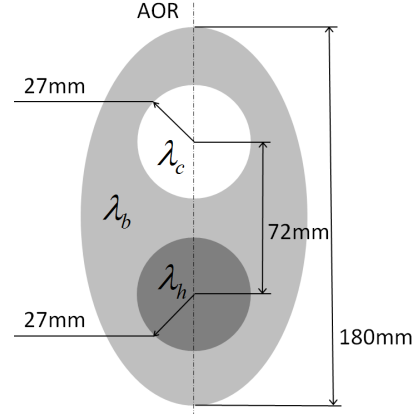

(a)

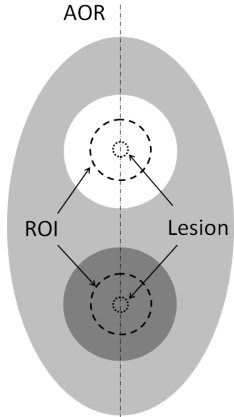

(b) Fig. 2. (a) The central profile of the contrast phantom used in volume imaging.
The ratio of activity $\lambda_{h}: \lambda_{b}: \lambda_{c}=8: 2: 1 . \lambda_{b}$ was $1.286 \times 10^{4} \mathrm{~Bq} / \mathrm{cm}^{2}$ for planar imaging and $7.144 \times 10^{4} \mathrm{~Bq} / \mathrm{cm}^{3}$ for volume imaging. (b) ROIs and lesions, with a diameter of $14.4 \mathrm{~mm}$ and $3.6 \mathrm{~mm}$, respectively.

The $\mathrm{PH}$ and the RS collimation systems were compared by calculating the gain (RS over $\mathrm{PH}$ ) in all three figures of merit $\left(\mathrm{CNR}_{\mathrm{pix}}, \mathrm{CNR}_{\mathrm{ROI}}\right.$ and $\left.\mathrm{SNR}_{\mathrm{CHO}}\right)$ :

$$
\begin{aligned}
\text { Gain }_{\mathrm{pix}} & =\mathrm{CNR}_{\mathrm{pix}}^{\mathrm{RS}} / \mathrm{CNR}_{\mathrm{pix}}^{\mathrm{PH}} \\
\text { Gain }_{\mathrm{ROI}} & =\mathrm{CNR}_{\mathrm{ROI}}^{\mathrm{RS}} / \mathrm{CNR}_{\mathrm{ROI}}^{\mathrm{PH}} \\
\text { Gain }_{\mathrm{CHO}} & =\mathrm{SNR}_{\mathrm{CHO}}^{\mathrm{RS}} / \mathrm{SNR}_{\mathrm{CHO}}^{\mathrm{PH}}
\end{aligned}
$$

With the definitions above, the trends of these gains as a function of various phantom parameters were investigated.

\section{NUMERICAL STUDY}

The comparative study of the two collimator systems was performed in both planar imaging and volume imaging. A $128 \times 128$ detector array was modeled for the $\mathrm{PH}$ and the RS collimator systems. The size of the collimator holes and the gap between the adjacent slat septa were both $1.8 \mathrm{~mm}$. The width of the detector $(W)$ was $230.4 \mathrm{~mm}$. The height of the collimator septa was $35.5 \mathrm{~mm}$ and $50 \mathrm{~mm}$ for the $\mathrm{PH}$ and the RS collimator, respectively. The ratio of the septa height ( $\mathrm{PH}$ over RS) was $\sqrt{2}$ according to $[9]^{3}$. For planar RS imaging and volume imaging, 100 rotation/spinning angles were equally distributed over 360 degrees. The digitized acquisition model and the geometric efficiency of the two systems were the same as described in [9]. The acquisition time was 40 minutes in both imaging modes.

The (central axial slice of the) contrast phantom used in planar (volume) imaging is shown in Fig. 2(a). The phantom consisted of an ellipse (ellipsoid) and two circles (spheres), representing the background, a hot organ and a cold organ, respectively. The uptake in these three parts was symbolized as $\lambda_{b}, \lambda_{h}$ and $\lambda_{c}$. The phantom was positioned in the center of the field of view, with its long axis coinciding with the rotation axis of the detector heads. The long and the short axis of the

\footnotetext{
${ }^{3}$ In [9], it was found that $\sigma_{p}^{o p t}=\sqrt{2} \sigma_{r}^{o p t}$, where $\sigma_{p}^{o p t}$ and $\sigma_{r}^{o p t}$ are the optimal collimator apertures of the $\mathrm{PH}$ and the RS system, respectively. For the same object-to-detector distance and the same pixel size, we have $\sigma_{p}^{o p t} / \sigma_{r}^{o p t}=$ $h_{r}^{o p t} / h_{p}^{o p t}=\sqrt{2}$, where $h_{r}^{o p t}$ and $h_{p}^{o p t}$ are the optimal septa heights of the $\mathrm{RS}$ and the $\mathrm{PH}$ collimator, respectively.
}

phantom were $180 \mathrm{~mm}$ and $108 \mathrm{~mm}$, respectively. The radius of each organ was $27 \mathrm{~mm}$ and the distance between the centers of the two organs was $72 \mathrm{~mm}$. The ratio of the activities $\lambda_{h}: \lambda_{b}: \lambda_{c}$ was $8: 2: 1$. The background activity $\lambda_{b}$ was $1.286 \times 10^{4} \mathrm{~Bq} / \mathrm{cm}^{2}$ for planar imaging and $7.144 \times 10^{4} \mathrm{~Bq} / \mathrm{cm}^{3}$ for volume imaging. The phantom was discretized with a pixel size of $0.75 \mathrm{~mm}$, and was reconstructed with a pixel size of $1.8 \mathrm{~mm}$.

The pixels of interest were chosen to be the central points of the two organs. Both the ROIs and the lesions were circular and located at the center of the organs (see Fig. 2(b)). The ROIs had a diameter of $14.4 \mathrm{~mm}$ and had the same activity as the organ, while the lesions had a diameter of $3.6 \mathrm{~mm}$ and the lesion-to-organ contrast was 2.

For the calculation of $\mathrm{SNR}_{\mathrm{CHO}}$, a difference-of-Gaussians (DOG) model with 3 channels was applied as the observer model. The channels were defined as the differences of pairs of a set of four 2-dimensional Gaussians. The Gaussians had a mean of 0 and standard deviation of $(2 d \sqrt{\pi})^{-1}$ where $d=$ $0.573,0.995,1.592$ and 2.653 . This channel model is the same as in [13].

In volume imaging, the attenuation within the phantom and the depth-dependent collimator blurring were modeled. The attenuation coefficient $\mu$ in the background and the hot region was $0.012 \mathrm{~mm}^{-1}$ and that in the cold organ was $0.0045 \mathrm{~mm}^{-1}$. Scatter and collimator septa penetration were not simulated in this study.

\section{A. Comparison between PH and RS}

We compared the two systems at a fixed spatial resolution of $10.8 \mathrm{~mm}$ FWHM. The distance between the detector plane and the center of the phantom was $100 \mathrm{~mm}$ and $150 \mathrm{~mm}$ in planar imaging and volume imaging, respectively.

We aimed to compare the two collimator systems using three FOMs and with respect to three phantom parameters, i.e, the gains in $\mathrm{CNR}_{\text {pix }}, \mathrm{CNR}_{\mathrm{ROI}}$ and $\mathrm{SNR}_{\mathrm{CHO}}$ were computed as a function of phantom size, organ contrast and the organ size.

1) Phantom Size: The phantom was globally scaled by a factor of $0.3,0.6,0.9$ and 1.2 , corresponding to a coverage of $2.5 \%$ to $40 \%$ of the detector area. The center of the phantom always coincided with the center of the image space.

2) Organ Contrast: The contrast ratio $\lambda_{h}: \lambda_{b}$ was varied from $1: 16$ to $16: 1$. The rest of the phantom parameters, including $\lambda_{b}$ and $\lambda_{c}$, were kept the same as described in Fig. 2.

3) Organ Size: The diameter of the each organ was scaled by a factor of $0.4,0.6,0.8$ and 1.0. The scaling was applied to only one organ at a time. The dimension of the phantom and the contrast $\lambda_{h}: \lambda_{b}: \lambda_{c}$ stayed the same as in Fig. 2.

\section{B. Validation}

The analytical method was verified for each data point in IIIA for planar imaging. In order to estimate how much postsmoothing we need to apply to the reconstruction to achieve the target resolution, we reconstructed the noiseless sinogram of the phantom with and without an impulse at the pixel of 


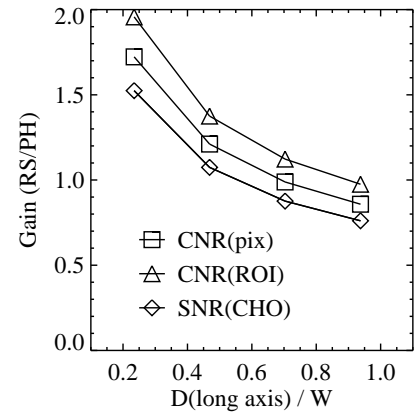

(a)

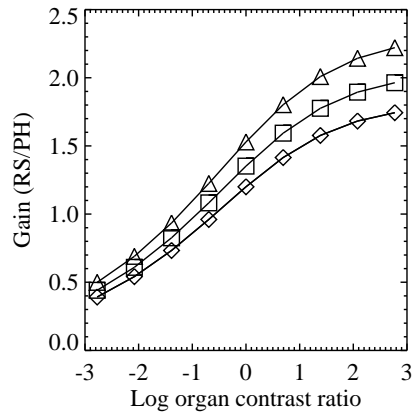

(b)

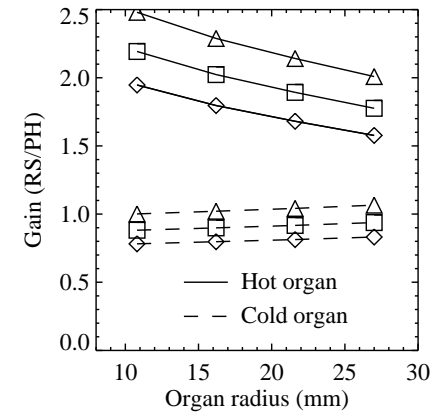

(c)

Fig. 3. Comparison results in planar imaging. (a) Gains $(\mathrm{RS} / \mathrm{PH})$ as a function of phantom size with $D$ the long axis of the phantom. The results are presented for the pixel/ROI/lesion in the cold organ. (b) Gains (RS/PH) as a function of $\ln \left(\lambda_{h} / \lambda_{b}\right)$, (c) Gains (RS/PH) as a function of organ size.

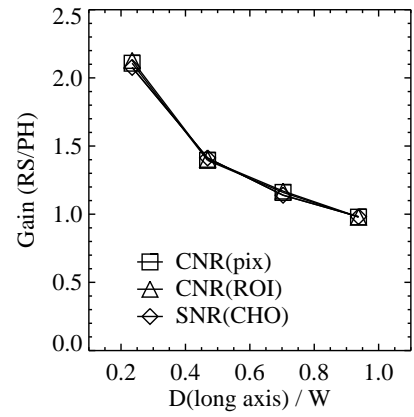

(a)

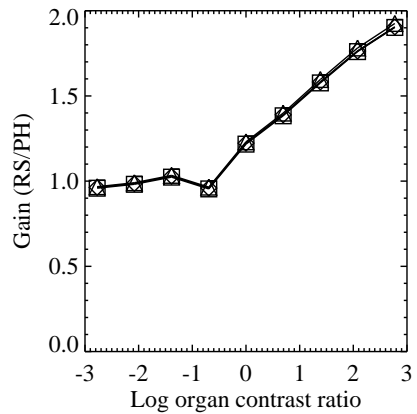

(b)

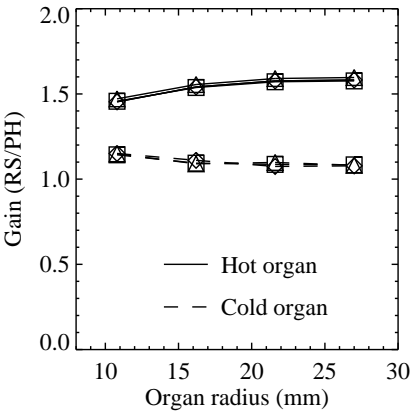

(c)

Fig. 4. Comparison results in volume imaging. (a) Gains (RS/PH) as a function of phantom size with $D$ the long axis of the phantom. The results are presented for the pixel/ROI/lesion in the cold organ. (b) Gains (RS/PH) as a function of $\ln \left(\lambda_{h} / \lambda_{b}\right)$, (c) Gains (RS/PH) as a function of organ size.

interest. The LLIR was then obtained by subtracting these two reconstructions. Based on the shape of LLIR, the postsmoothing filter was derived and applied to each of the noisy reconstruction images.

For the variance calculation, 200 noise realizations were simulated and reconstructed. For the PH system, we performed standard MLEM reconstruction with 100 iterations. For the RS system, we applied ordered subset expectation maximization (OSEM) [25] with a reducing number of subsets, i.e., $20 \times 20$, $20 \times 10,20 \times 5,20 \times 2,20 \times 1$ (number of global iterations $\times$ number of subsets $)^{4}$. The values of $\mathrm{CNR}_{\mathrm{pix}}, \mathrm{CNR}_{\mathrm{ROI}}$ and $\mathrm{SNR}_{\mathrm{CHO}}$ calculated from the multiple noisy reconstructions were used as the reference values and compared to the values yielded by the analytical method.

\section{Optimal Aperture}

In [9], it was found that $\mathrm{CNR}_{\text {pix }}$ is influenced by the collimator aperture, and this influence differs from collimator to collimator. For a given target resolution and a certain collimator

\footnotetext{
${ }^{4}$ With this scheme, the OSEM reconstruction will start with 20 iterations over 20 subsets, then the image will be further updated with another 20 iterations over 10 subsets, and so on. The reconstruction procedure will be ended by applying 20 normal MLEM reconstructions (= OSEM with 1 subset). The purpose is to improve the convergence of the OSEM reconstruction [25].
}

type, there exists an optimal collimator aperture which yields the maximal $\mathrm{CNR}_{\mathrm{pix}}$ in the investigated pixel. In the framework of [9], we have only derived the optimal PH/RS aperture for $\mathrm{CNR}_{\text {pix }}$ and $\mathrm{CNR}_{\mathrm{ROI}}$ based on uniform phantoms. In this paper, we used the same approach described in [9] to calculate and compare the $\mathrm{PH} / \mathrm{RS}$ apertures which were optimized for $\mathrm{CNR}_{\text {pix }}, \mathrm{CNR}_{\mathrm{ROI}}$ and $\mathrm{SNR}_{\mathrm{CHO}}$ respectively based on the $2 \mathrm{D}$ contrast phantom.

\section{RESUlts}

\section{A. Comparison between $P H$ and $R S$}

Using the phantom of Fig. 2, we calculated the gains in all three FOMs with various phantom settings. In each setting, we only changed one phantom parameter, and fixed all the other parameters to those indicated in Fig. 2. The comparison results are shown in Fig. 3 and Fig. 4, where the gains in $\mathrm{CNR}_{\text {pix }}, \mathrm{CNR}_{\mathrm{ROI}}$ and $\mathrm{SNR}_{\mathrm{CHO}}$ are plotted with a square, triangle and diamond, respectively. The absolute values of most of the FOMs are not shown in the figures but briefly discussed in the text.

1) Phantom Size: Fig. 3(a) and Fig. 4(a) show the results in the cold organ. All the gains decrease with increasing phantom 


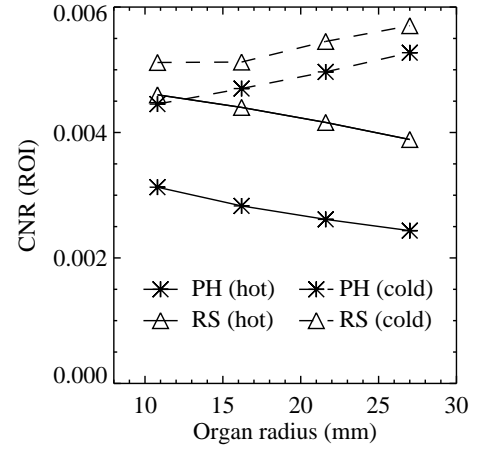

Fig. 5. Absolute values of $\mathrm{CNR}_{\mathrm{ROI}}$ in Fig. 4(c) as a function of organ size The solid/dashed line represents the ROI in the hot/cold organ, respectively. The asterisk and triangle represent $\mathrm{PH}$ and RS, respectively.

size $^{5}$. The RS collimator outperforms $\mathrm{PH}$ when the phantom was small, however the advantage is lost when the phantom covers up to $40 \%$ of the detector area. In the hot organ, the RS collimator is always superior to the $\mathrm{PH}$ collimator, where the gains were about 1.9 (in planar imaging) and 1.4 (in volume imaging) times higher than those in the cold organ (not shown in the figures).

For planar imaging with the PH collimator, the absolute value of each FOM is the same for all phantom sizes, whereas in other cases, all figures of merit decrease with increasing phantom size.

2) Organ Contrast: As shown in Fig. 3(b) and Fig. 4(b), all gains go up with increasing organ contrast. In planar imaging, the $\mathrm{PH}$ collimator is superior to the RS collimator in the cold region, while in volume imaging, the two collimators have similar performance in low contrast $($ cold) areas $($ gain $\approx 1)$. In both imaging modes, the absolute values of $\mathrm{CNR}_{\text {pix }}$ and $\mathrm{CNR}_{\mathrm{ROI}}$ decrease with increasing organ activity with either of the collimator systems. The CNR values with the $\mathrm{PH}$ collimator are more affected by the change in the contrast. For lesion detection, $\mathrm{SNR}_{\mathrm{CHO}}$ goes up with both collimators when the uptake of the organ increases.

3) Organ Size: Fig. 3(c) and Fig. 4(c) show different trends of the gains in planar and volume imaging. However, one should note that the absolute values of $\mathrm{CNR}_{\text {pix }}$ and $\mathrm{CNR}_{\mathrm{ROI}}$ obtained with both collimator systems are always decreased/increased in the hot/cold organ when the organ size increases. As an example, $\mathrm{CNR}_{\mathrm{ROI}}$ in volume imaging are plotted in Fig. 5. In general, the RS collimator outperforms the $\mathrm{PH}$ collimator for hot spot imaging (gains $>1$ ), and the results with the two collimators are very similar for cold spot imaging $($ gain $\approx 1)$.

${ }^{5}$ Here we use the ratio of the long axis of the phantom and the detector width $(D / W)$ as the measure of the phantom size. When the phantom is globally scaled by a factor of $0.3,0.6,0.9$ and 1.2 , the corresponding value of $D / W$ is $0.23,0.47,0.70$ and 0.94 , respectively.

\section{B. Validation}

The validation for $\mathrm{CNR}_{\mathrm{pix}}, \mathrm{CNR}_{\mathrm{ROI}}$ and $\mathrm{SNR}_{\mathrm{CHO}}$ was done for each data point in Fig. 3. The results are shown in Fig. 6. The asterisk and triangle represent $\mathrm{PH}$ and RS, respectively. The values calculated using the analytical method are plotted with respect to the reference values derived from the reconstructions. The dashed line and the dotted line represent the $68.3 \%$ and $95.4 \%$ confidence level of the reference values, respectively. These confidence levels are estimated on the assumption that the relative error on the standard deviation is Gaussian with a standard deviation of $\sqrt{1 /(2(N-1))}$, where $N$ is the number of noise realizations (With $N=200$, the resulting relative error on the standard deviations is around 5\%). Based on the analysis shown in Table I, we can see that the approximated values are in good agreement with their corresponding reference values.

\section{Optimal Aperture}

Fig. 7 gives the results about the optimal aperture. Here we again use the square, the triangle and the diamond to represent $\mathrm{CNR}_{\mathrm{pix}}, \mathrm{CNR}_{\mathrm{ROI}}$ and $\mathrm{SNR}_{\mathrm{CHO}}$. The solid and dashed lines indicate $\mathrm{PH}$ and RS, respectively. As shown in Fig. 7(a), each FOM yields its own optimal aperture sets. Although the optimal apertures are different for different FOMs, the ratio of the optimal apertures (PH/RS) is always close to $\sqrt{2}$ (Fig. 7(b)), a factor that we derived analytically in [9].

\section{Discussion}

For the comparative study, an analytical method was used to estimate three figures of merit. Although the analytical method was only validated in planar imaging, we believe that it will be also valid for volume imaging due to its capability of accommodating the shift-variant system response (validation done for multi-pinhole SPECT systems [20]) and the attenuation within the phantom (validation done for time-of-flight positron emission tomography [21]).

Our analytical method assumes that the reconstruction is fully converged. To this end, the reconstructed images used for validation should be iterated long enough to reach their convergences. To verify this, we applied twice the number of iterations for both $\mathrm{PH}$ and $\mathrm{RS}$ in the first comparison settings (with various phantom size), and the validation results were hardly changed (not shown in this paper). Therefore, we believe that the iteration scheme used in the validation study is sufficient to generate converged reconstructions.

Due to the increased noise propagation, the reconstruction of the RS data needs more post-smoothing than that of the $\mathrm{PH}$ data. Therefore, for a matched spatial resolution, the aperture of the RS collimator should be smaller than that of PH. Previously, it was found that the ratio of the collimator aperture (PH over RS) should be $\sqrt{2}$ when $\mathrm{CNR}_{\text {pix }}$ is used as the FOM [9]. Therefore, in this work, we applied the ratio of $\sqrt{2}$ in the collimator geometry setting, with which all the FOMs were estimated using contrast phantoms.

We also optimized the collimator apertures for each FOM individually. Interestingly, the rule of $\sqrt{2}$ remains valid for 


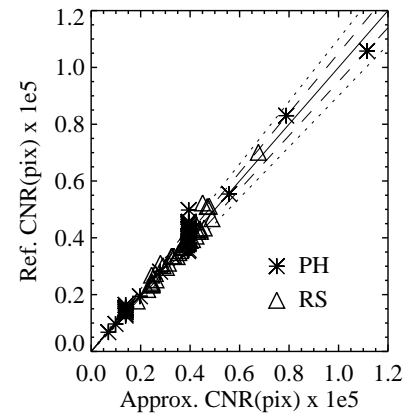

(a)

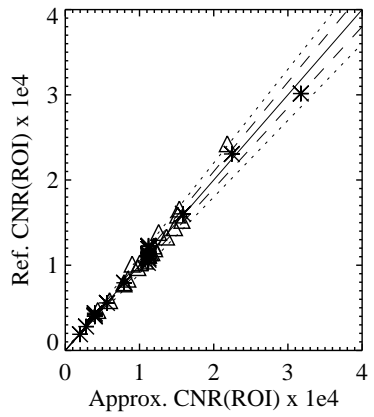

(b)

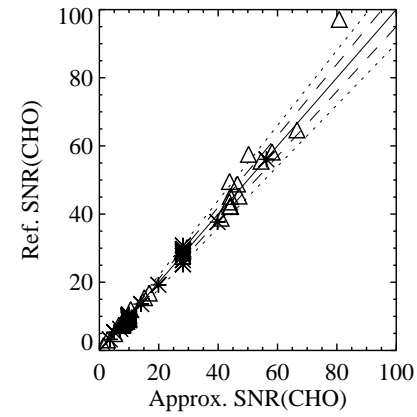

(c)

Fig. 6. The validation results of (a) $\mathrm{CNR}_{\text {pix }}$, (b) $\mathrm{CNR}_{\mathrm{ROI}}$, (c) $\mathrm{SNR}_{\mathrm{CHO}}$. The solid line indicates the perfect agreement, and the dashed and the dotted line indicate the $68.3 \%$ and $95.4 \%$ confidence level, respectively.

TABLE I

ANALYSIS OF THE VALIDATION RESULTS IN FIG. 6

\begin{tabular}{|c|c|c|c|c|c|c|}
\hline & \multicolumn{3}{|c|}{ PH } & \multicolumn{3}{c|}{ RS } \\
\hline FOM & CNR $_{\text {pix }}$ & CNR $_{\text {ROI }}$ & SNR $_{\text {cho }}$ & CNR $_{\text {pix }}$ & CNR $_{\text {ROI }}$ & SNR $_{\text {cho }}$ \\
\hline slope (least squares fitting) & 0.998 & 0.971 & 0.995 & 1.036 & 1.089 & 1.063 \\
\hline \% points in 68.3\% confidence level & $38.1 \%$ & $66.7 \%$ & $61.9 \%$ & $78.6 \%$ & $76.2 \%$ & $71.4 \%$ \\
\hline \% points in 95.4\% confidence level & $78.6 \%$ & $100 \%$ & $90.5 \%$ & $95.2 \%$ & $85.7 \%$ & $85.7 \%$ \\
\hline
\end{tabular}

$\mathrm{CNR}_{\mathrm{ROI}}$ and $\mathrm{SNR}_{\mathrm{CHO}}$ as shown in Fig. 7(b). Based on this finding, we propose to compare the $\mathrm{PH}$ and the RS systems with their collimator aperture ratio equal to $\sqrt{2}$, no matter which figure of merit is under investigation.

It was found that the absolute values of $\mathrm{CNR}_{\mathrm{pix}}$ and $\mathrm{CNR}_{\mathrm{ROI}}$ decreased with increasing organ-to-background ratio, while $\mathrm{SNR}_{\mathrm{CHO}}$ has the opposite trend. This can be explained as follows. To calculate $\mathrm{SNR}_{\mathrm{CHO}}$, the lesion has a fixed contrast relative to the organ activity, so it is hotter for hotter organs, and is easier to be detected by the observers. In contrast, for the calculation of $\mathrm{CNR}_{\text {pix }}$ and $\mathrm{CNR}_{\mathrm{ROI}}$, we applied a small intensity change in a pixel or a ROI, where the change is independent of the activity of the organs. As a result, it is more difficult to recover this small change with higher surrounding activity, resulting in a lower CNR.

As shown in Fig. 3 and Fig. 4, the gains (RS over $\mathrm{PH}$ ) in all three FOMs give the same trends in the system comparison. It means that when the phantom parameter varies, the relative performance of the two systems will be influenced in the same way for different imaging tasks, i.e., if one system is superior to the other system for the noise property in a ROI, probably it will also win in the aspect of lesion detectability.

From Fig. 3 and Fig. 4, we can conclude that the RS collimator outperforms the $\mathrm{PH}$ collimator for hot spot imaging (for instance, 1.5-2 times improvement with the given phantom size). For cold organ and lesions, $\mathrm{PH}$ is superior to RS in planar imaging, whereas the two collimator systems have very similar performance in volume imaging. Note that these conclusions are drawn with a phantom covering up to $40 \%$ of the detector area, and the phantom is not truncated by the detector. When the phantom is larger than that, the performance of the $\mathrm{PH}$ collimator with respect to the RS collimator will be better, especially for cold spot imaging.

Our conclusion about the planar imaging is in agreement with [11], where the phantom also covers about $40 \%$ of the detector area. However, in volume imaging, the gain of using the RS collimator in our study is very different from that proposed in [12], where it was found that the RS collimator is 2-3 times better than the $\mathrm{PH}$ in hot spot imaging, and more than 4 times better in cold spot imaging. One of the main reasons is that the collimator apertures in [11], [12] were the same for both collimators, whereas in our study we applied the optimal collimator aperture for each system to ensure a fair comparison. The discrepancy could also be partially explained by the influence of photon scatter, which was modelled in [12] but not in this paper.

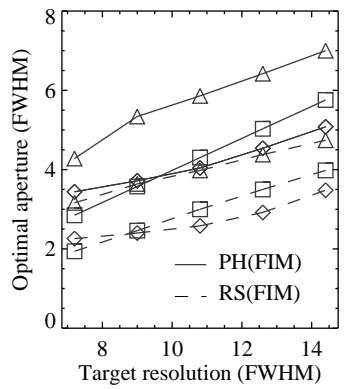

(a)

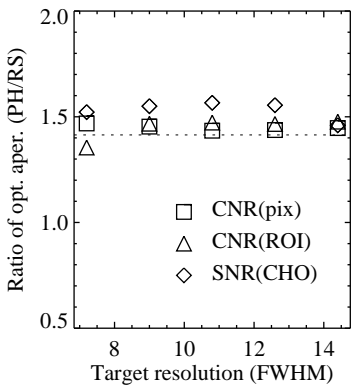

(b)
Fig. 7. (a) Optimal collimator apertures as function of the target resolution. (b) Ratio of the optimal aperture. The dotted line represents the value of $\sqrt{2}$. 
The dimensions of the phantom used in this study were relatively small compared to the human size. Actually, the relative performance of the two collimators depends on the size of the object with respect to the detector area [9]. Therefore, all the conclusions should hold if the whole geometry is scaled to a clinically relevant dimension. The RS collimator is preferred when focused uptake is expected, for instance in human brain imaging with a highly specific SPECT tracer. However, if there is activity outside the FOV, the RS data will be contaminated by these activities, leading to increased variance and truncation artefacts in the reconstructed image. The degradation of the image quality depends on the amount of data truncation. Therefore, for whole body imaging the PH collimator is still highly recommended.

One of the main conclusions is that the RS collimator outperforms $\mathrm{PH}$ for small object imaging. One may argue that to image very small objects, collimators with converging geometries, such as a fan-beam/cone-beam collimator or a pinhole collimator should be considered, since they exploit the detection area in a more efficient way. In fact, it is expected that the gain of using a plane-integral geometry instead of using a line-integral geometry will be applicable for converging collimators as well. It means that instead of using a fanbeam collimator, it is probably better to apply a RS collimator with converging slat septa, or that we could consider taking a rotating slit collimator rather than a pinhole collimator, with their geometries optimized for a certain target resolution.

In this study, photon scatter and septa penetration were not modelled. The degradation of the image quality due to he contamination from the scattered and penetrated photons depends on the photon energy, i.e., the contamination are much stronger with high energy isotopes (e.g. $\mathrm{I}^{123}$ and $\mathrm{I}^{131}$ ) than with low energy isotopes (e.g. Tc ${ }^{99 m}$ ). Due to its high geometric efficiency, the RS collimator will benefit from a lower contribution of penetrated photons than the $\mathrm{PH}$ collimator. This will result in a 10\%-20\% improved contrast recovery for the RS collimator with $\mathrm{I}^{123}$ and $\mathrm{I}^{131}$ [26]. Combining this finding with our results, we can conclude that, as long as the object to be imaged is smaller than the detector area, the RS collimator is recommended, especially for imaging with high energy emitters.

\section{CONCLUSION}

We applied three FOMs $\left(\mathrm{CNR}_{\text {pix }}, \mathrm{CNR}_{\mathrm{ROI}}\right.$ and $\left.\mathrm{SNR}_{\mathrm{CHO}}\right)$ to compare the $\mathrm{PH}$ and the RS collimator systems based on digital contrast phantoms. The gains of all FOMs show the same trends in the system comparison. RS is always superior to $\mathrm{PH}$ for hot spot imaging. For cold spot and lesions, $\mathrm{PH}$ outperforms RS in planar imaging, while the two collimator systems have very similar performance in volume imaging. For system optimization, the optimal collimator apertures depend on the FOM to be optimized, however the ratio of the optimal aperture $(\mathrm{PH} / \mathrm{RS})$ is always around $\sqrt{2}$, a factor that we derived analytically in [9].

\section{REFERENCES}

[1] D. Gagnon, G. L. Zeng, J. M. Links, J. J. Griesmer, and F. C. Valentino, "Design considerations for a new solid-state gamma-camera SOLSTICE." IEEE Nuclear Science Symposium Conference Record, pp. 1156-1160, 2002.

[2] S. Webb, M. A. Flower and R. J. Ott, "Geometric efficiency of a rotating slit-collimator for improved planar gamma-camera imaging." Phys. Med. Biol., 38, pp. 627-638, 1993.

[3] S. Webb, D. M. Binnie, M. A. Flower and R. J. Ott, "Monte Carlo modelling of the performance of a rotating slit-collimator for improved planar gamma camera imaging." Phys. Med. Biol., 37(5), pp. 1095-1108, 1992.

[4] S. Vandenberghe, R. Van Holen, S. Staelens and I. Lemahieu, "System characteristics of SPECT with a slat collimated strip detector." Phys. Med. Biol., 51, pp. 391-405, 2006.

[5] M. A. Lodge, D. M. Binnie, M. A. Flower and S. Webb, "The experimental evaluation of a prototype rotating slat collimator for planar gamma camera imaging." Phy. Med. Biol., 40, pp. 427-448, 1995.

[6] M. A. Lodge, S. Webb, M. A. Flower and D. M. Binnie, "A prototype rotating slat collimator for single photon emission computed tomography." IEEE Trans. Med. Imag., 15(4), pp. 500-511, 1996.

[7] B. Zhang and G. L. Zeng, "Study of noise propagation and the effects of insufficient numbers of projection angles and detector samplings for iterative reconstruction using planar-integral data." Med. Phys., 33(9), pp. 3124-3134, 2006.

[8] W. Wang, W. Hawkins and D. Gagnon, "3D RBI-EM reconstruction with spherically-symmetric basis function for SPECT rotating slat collimator." Phys. Med. Biol., 49, pp. 2273-2292, 2004.

[9] L. Zhou, M. Defrise, K. Vunckx, and J. Nuyts. "Comparison between parallel hole and rotating slat collimation: analytical noise propagation models." IEEE Trans. Med. Imag., 29(12), pp. 2038-2052, 2010.

[10] C. Byrne, "Accelerating the EMML algorithm and related iterative algorithms by rescaled block-iterative methods." IEEE Trans. Image Proc., 7, pp. 100-109, 1998.

[11] R. Van Holen, S. Vandenberghe, S. Staelens and I. Lemahieu, "Comparing planar image quality of rotating slat and parallel hole collimation: influence of system modeling." Phys. Med. Biol., 53, pp. 1989-2002, 2008.

[12] R. Van Holen, S. Staelens and S. Vandenberghe, "Tomographic image quality of rotating slat versus parallel hole-collimated SPECT." Phys. Med. Biol., 56, pp. 7205-7222, 2011.

[13] H. C. Gifford, M. A. King, D. J. de Vries and E. J. Soares, "Channelized Hotelling and human observer correlation for lesion detection in hepatic SPECT imaging." J. Nucl. Med., 41(3), pp. 514-521, 2000.

[14] K. J. Myers and H. H. Barrett, "Addition of a channel mechanism to the ideal-observer model." J. Opt. Soc. Am. A, 4(12), pp. 2447-2457, 1987.

[15] T. K. Narayan and G. T. Herman. "Prediction of human observer performance by numerical observers: an experimental study." J. Opt. Soc. Am. A, 16(3), pp. 679-693, 1999.

[16] S. D. Wollenweber, B. M. W. Tsui, D. S. Lalush, E. C. Frey, K. J. LaCroix, and G. T. Gullberg, "Comparison of Hotelling observer models and human observers in defect detection from myocardial SPECT imaging." IEEE Trans. Nucl. Sci., 46(6), pp. 2098-2103, 1999.

[17] J. A. Fessler, "Mean and variance of implicitly defined biased estimators (such as penalized maximum likelihood): applications to tomography." IEEE Trans. Image Processing, 5(3), pp. 493-506, 1996.

[18] J. A. Fessler and W. L. Rogers, "Spatial resolution properties of penalized likelihood image reconstruction: space-invariant tomographs." IEEE Trans. Image Proc., 5(9), pp. 1346-1358, 1996.

[19] J. Qi and R. M. Leahy, "A theoretical study of the contrast recovery and variance of MAP reconstructions from PET data." IEEE Trans. Med. Imaging, 18(4), pp. 293-305, 1999.

[20] K. Vunckx, D. Bequé, M. Defrise and J. Nuyts, "Single and multipinhole collimator design evaluation method for small animal SPECT." IEEE Trans. Med. Imaging, 27(1), pp. 36-46, 2008.

[21] K. Vunckx, L. Zhou, S. Matej, M. Defrise and J. Nuyts, "Fisher information-based evaluation of image quality for time-of-flight PET." IEEE Trans. Med. Imaging, 29(2), pp. 311-321, 2010.

[22] J. Qi, "Analysis of lesion detectability in Bayesian emission reconstruction with nonstationary object variability." IEEE Trans. Med. Imaging, 23(3), pp. 321-329, 2004.

[23] A. Yendiki, J. A. Fessler, "Analysis of observer performance in knownlocation tasks for tomographic image reconstruction." IEEE Trans. Med. Imaging, 25(1), pp. 28-41, 2006. 
[24] A. Yendiki, J. A. Fessler, "Analysis of observer performance in unknownlocation tasks for tomographic image reconstruction." J. Opt. Soc. Sm. A Optic Image Sci. Vis., 24(12), pp. B99-B109, 2007.

[25] H. M. Hudson and R. S. Larkin, "Accelerated image reconstruction using ordered subsets of projection data." IEEE Trans. Med. Imag., 13(4), pp. 601-609, 1994.

[26] R. Van Holen, S. Staelens and S. Vandenberghe, "SPECT imaging of high energy isotopes and isotopes with high energy contaminants with rotating slat collimators." Med. Phys., 36(9), pp. 4257-4267, 2009. 